\title{
L'accessibilité financière à la complémentaire santé en France : les résultats de l'enquête Budget de Famille 2006
}

\author{
Florence Jusot, Clémence Perraudin et Jérôme Wittwer*
}

\begin{abstract}
La Sécurité sociale prenant en charge 75,8 \% des dépenses de soins en France, l'accès aux soins repose en partie sur la possession d'un contrat de couverture complémentaire. Des dispositifs ont été mis en place par les autorités publiques afin d'améliorer l'accès des ménages les plus pauvres à une couverture complémentaire comme la Couverture maladie universelle complémentaire (CMU-C) et l'Aide complémentaire santé (ACS). Cependant, en dépit de ces dispositifs, subsiste-t-il des barrières financières limitant l'accessibilité à la complémentaire santé ? La persistance de personnes non couvertes ou faiblement couvertes peut-elle s'expliquer par les difficultés financières des ménages les plus modestes?
\end{abstract}

À partir de l'enquête Budget de Famille 2006 de l'Insee, cette étude décrit la distribution selon le niveau de revenu du taux de couverture par une complémentaire santé puis évalue l'accessibilité financière d'une complémentaire santé par analyse de la distribution des dépenses engagées par les ménages pour en bénéficier. Enfin sont étudiés les déterminants du recours à une complémentaire santé ainsi que ceux en jeu dans le niveau de dépenses engagées pour cet achat.

Les résultats confirment l'existence d'un obstacle financier à l'accès à la complémentaire santé en France. Le taux de couverture et le montant des dépenses consacrées augmentent fortement avec le niveau de revenu. Néanmoins, les ménages les plus modestes consacrent à l'acquisition d'un contrat de couverture complémentaire respectivement $8,5 \%$ et $10,7 \%$ de leur revenu disponible selon qu'ils sont couverts par un contrat individuel ou par le biais de leur employeur, alors que les plus riches n'y consacrent respectivement que $2,3 \%$ et 3,3\%. L'assurance complémentaire peut être jugée comme non accessible financièrement pour $18,5 \%$ de la population française et pour $10,3 \%$ de la population non couverte par la CMU-C, dans la mesure où son achat les conduit à tomber en dessous du seuil de pauvreté.

* Clémence Perraudin : CERMES3, CNRS UMR 8211 - Inserm U988, Villejuif, France

Florence Jusot: PSL, Université Paris-Dauphine, LEDa-LEGOS, Paris, France.

Jérôme Wittwer : Université Bordeaux Segalen, ISPED et PSL, Université Paris-Dauphine, LEDa-LEGOS.

Les auteurs remercient vivement Anne Legal pour son aide dans le traitement des données, ainsi que Sandy Tubeuf, Thomas Buchmueller, les participants aux $30^{\circ}$ Journées des économistes de la santé Français (Université Paris-Dauphine, décembre 2008), au LEGOS-IRDES Health Insurance Workshop (Université Paris-Dauphine, mai 2010), et à la $8^{\circ}$ European Conference on Health Economics (Helsinki, juillet 2010), pour leurs commentaires et suggestions. 
L e système français de financement des dépenses de soins a la particularité de laisser à la charge du patient une partie du coût des soins. Globalement, la Sécurité sociale prend en charge les trois quarts de la consommation de biens et soins médicaux $(75,8 \%$ en 2010$)$ mais la part laissée à la charge des patients est importante pour certains types de soins, tels que pour les médicaments, les lunettes et autres types de prothèses, et les soins dentaires (Fenina et al., 2011). Pour se couvrir contre ce risque financier, les individus peuvent recourir au marché de l'assurance santé complémentaire sur lequel les organismes de complémentaire santé - mutuelles, assureurs ou instituts de prévoyance - proposent, moyennant le paiement de primes, des contrats dits individuels prenant en charge dans des proportions et des modalités variables des dépenses non couvertes par l'Assurance maladie. Par ailleurs, certains salariés bénéficient d'une couverture complémentaire d'entreprise partiellement prise en charge par leur employeur dans le cadre de contrats dits collectifs. Ce second type de contrat concerne en premier lieu les cadres et les salariés des grandes entreprises (Guillaume et Rochereau, 2010).

Le recours à une complémentaire santé dans la population reste un enjeu de santé publique important dans la mesure où l'absence de couverture complémentaire et les restes à charge conséquents qui en découlent constituent un obstacle au recours aux soins, tout particulièrement parmi les ménages les plus pauvres (Boisguérin et al., 2010 ; Buchmueller et al., 2004 ; Raynaud, 2005 ; Kambia-Chopin et al., 2008a ; Jusot et Wittwer, 2009 ; Perronnin et al., 2011). Plusieurs dispositifs déjà mis en place par les autorités publiques, comme la couverture maladie universelle complémentaire $(\mathrm{CMU}-\mathrm{C})^{1}$ et l'aide complémentaire santé $(\mathrm{ACS})^{2}$, témoignent ainsi de leur préoccupation d'éliminer les barrières financières à l'accès aux soins. Si l'objectif visé par ces politiques est l'amélioration de l'accès financier aux soins des plus pauvres au travers d'une couverture complète de cette population, il convient de s'interroger sur l'atteinte de ces objectifs et plus spécifiquement, sur le maintien de barrières financières au recours à la complémentaire santé.

À partir de l'enquête Budget de Famille 2006 de l'Insee (BdF06, cf. encadré 1), cet article propose d'étudier l'accès à la complémentaire santé en France et d'analyser les barrières financières pouvant limiter l'accessibilité à la complémentaire santé des ménages les plus modestes.

Le premier apport de cette étude consiste en une utilisation de l'enquête Budget de Famille, très rarement utilisée dans cette perspective. Cette étude fournit ainsi des éléments quantitatifs nouveaux venant compléter les connaissances sur le taux de couverture et sa distribution, qui sont à ce jour uniquement établies à partir des enquêtes Santé Protection Sociale (ESPS) de l'Irdes (Arnould et Vidal, 2008 ; KambiaChopin et al., 2008a ; 2008b ; Perronnin et al., 2011) et de l'enquête décennale Santéde l'Insee (Marical et de Saint Pol, 2007).

Cette enquête présente en outre deux avantages de taille. Le premier est d'apporter des informations particulièrement précises sur les revenus des ménages, fournissant ainsi des données plus fiables que dans la plupart des enquêtes menées en population générale. Cette enquête offre alors la possibilité d'étudier de manière précise la distribution selon le niveau de revenu du taux de couverture par une complémentaire santé. Le deuxième avantage de cette enquête est de répertorier de manière unique en France l'ensemble des consommations des ménages, et de renseigner non seulement le montant de la prime d'assurance payée directement par les ménages aux organismes d'assurance complémentaire afin d'être couverts dans le cadre d'un contrat individuel, mais également le montant des prélèvements à la source effectués par les employeurs au titre de la participation des salariés à la complémentaire santé d'entreprise, ce qui n'est pas recueilli dans les autres enquêtes. Cette seconde spécificité offre alors la possibilité d'étudier précisément la distribution du montant de l'ensemble des dépenses de complémentaire santé engagées par

1. Au 31 décembre 2010, 4319165 personnes bénéficiaient gratuitement d'une couverture en raison de la faiblesse de leurs revenus par le biais de la couverture maladie universelle complémentaire (CMU-C) (Fonds CMU, 2011). Ce dispositif, mis en place en 2000, propose une couverture à $100 \%$ et sans avance de frais pour un assez large panier de soins pour les personnes ayant un niveau de vie inférieur à un certain seuil. En 2006, ce dispositif concernait les individus résidant en France de façon stable et régulière et disposant d'un niveau de vie inférieur à 598,23 euros par mois pour une personne seule, soit $7 \%$ de la population française (Arnoud et Vidal, 2008).

2. L'aide complémentaire santé (ACS), appelée parfois chèque santé, a été mise en place en 2005 et concernait 632785 personnes au 31 décembre 2010 (Fonds CMU, 2011). II s'agit d'une aide financière correspondant à environ $50 \%$ du prix des contrats de complémentaire proposée aux personnes dont le niveau de vie est compris entre le seuil d'éligibilité à la CMUC et ce plafond majoré de 15 \% jusqu'au 31 décembre 2006, $20 \%$ jusqu'au 31 décembre 2010, 26 \% jusqu'au 31 décembre 2011 et $35 \%$ à partir du 1er janvier 2012. En 2006, $1 \%$ de la population française bénéficiait de cette aide (Arnould et Vidal, 2008) et le plafond s'établissait à 687,97 euros par mois pour une personne seule (Fonds CMU, 2008). 
les ménages et de s'interroger sur l'accessibilité financière à une complémentaire santé, en adoptant la méthodologie proposée par Bundorf et Pauly (2006).

Cette étude propose enfin d'approfondir les connaissances sur le rôle du revenu dans la demande de complémentaire santé. Un nombre très réduit de travaux ont traité cette question en France. On relèvera cependant l'étude de Saliba et Ventelou (2007) centrée sur les déterminants de la détention d'une complémentaire santé à titre individuel et l'étude de Grignon et Kambia-Chopin $(2009$; 2010) centrée sur les déterminants du montant de la dépense de complémentaire santé à titre individuel parmi les personnes couvertes. À l'instar des travaux de Marquis et Long (1995) et Auerbach et Ohri (2006), réalisés sur données américaines, ils soulignent l'influence très nette du revenu sur la probabilité de détenir une complémentaire santé et l'absence de rôle de l'état de santé déclaré, alors même que les primes sont, en France, peu dépendantes du risque. Nous proposons ici une analyse conjointe des déterminants respectifs de la détention d'une complémentaire santé à titre individuel et de la dépense engagée pour sa souscription. L'utilisation d'un modèle Tobit généralisé permet ainsi d'étudier les spécificités de la décision de couverture par un contrat de complémentaire d'une part et du montant des dépenses de complémentaire santé. Cette dernière analyse permet alors de s'intéresser de façon indirecte aux déterminants du choix de la qualité d'une assurance santé puisque, même si l'enquête BDF06 ne contient pas d'informations précises sur les caractéristiques des contrats souscrits, le lien entre montant des primes et qualité de la couverture a été établi par ailleurs (Arnould et Vidal, 2008).

\section{Encadré 1 \\ MESURER LE TAUX DE RECOURS À LA COMPLÉMENTAIRE SANTÉ DANS L'ENQUÊTE BUDGET DE FAMILLE 2006 (BDF06)}

L'enquête Budget de famille 2006, réalisée par I'INSEE, porte sur un échantillon représentatif de 10236 ménages « ordinaires » résidant en France métropolitaine, correspondant à 25360 individus âgés de 18 ans et plus. L'objectif de cette enquête étant de mesurer le plus précisément possible les dépenses et les ressources des ménages résidant en France, ce sont donc les ressources sous toutes leurs formes qui sont enregistrées : revenus imposables ou non, prestations sociales, revenus du patrimoine, sommes provenant d'autres ménages, ressources exceptionnelles (loto, héritage, prime de licenciement, etc. ) ; ainsi que l'ensemble des dépenses (avec des informations sur leur montant et leur nature), dépenses qui sont par la suite ventilées dans une nomenclature détaillée d'environ 900 postes budgétaires. L'enquête se déroule en trois visites au cours desquelles les ménages et/ou les individus qui le composent répondent à trois questionnaires avec une question spécifique : « Avez-vous eu une dépense pour une mutuelle ou une assurance privée au cours des 12 derniers mois ? ». Par ailleurs, un carnet de dépenses est rempli par les ménages durant 15 jours, à partir duquel les dépenses annuelles sont calculées en multipliant par 26 les dépenses relevées.

Plusieurs informations sont disponibles dans l'enquête Bdf06 pour repérer le fait d'être couvert par une complémentaire santé ainsi que le montant des dépenses engagées pour un contrat de couverture complémentaire santé.

A l'échelle du ménage, tout d'abord, sont renseignées les dépenses engagées au cours des douze derniers mois par le ménage pour l'achat d'un contrat de couverture complémentaire auprès d'une mutuelle ou d'une assurance privée. Par ailleurs, chaque individu du ménage occupant un emploi de salarié déclare si son employeur a effectué au cours du dernier mois un prélèvement à la source sur son salaire pour l'achat d'une couverture complémentaire et le montant de ce prélèvement le cas échéant. Cette enquête permet donc de connaître à l'échelle des ménages le montant des dépenses engagées directement par les ménages ainsi que la somme des prélèvements effectués par les employeurs des différents membres du ménage pour l'achat d'un contrat de couverture complémentaire.

Nous considérons qu'un ménage est couvert par une complémentaire santé à titre onéreux si le ménage déclare avoir engagé des dépenses pour une mutuelle ou une assurance privée au cours des douze derniers mois et/ou si une personne au moins du ménage déclare qu'un prélèvement à la source sur le salaire a été effectué par l'employeur au cours du dernier mois pour une assurance ou une mutuelle complémentaire maladie.

Parmi les ménages couverts à titre onéreux, nous considérons que les ménages n'ayant déclaré que des dépenses engagées par eux-mêmes auprès d'un organisme complémentaire sont couverts dans le cadre d'un contrat individuel. Dans le cas où un prélèvement effectué par l'employeur est déclaré par l'un au moins des membres du ménage, nous supposons que le ménage est couvert par un contrat collectif, qu'une dépense auprès d'un organisme complémentaire ait été ou non par ailleurs déclarée au cours des douze derniers mois. Cette définition tend à considérer que les ménages de fonctionnaires couverts par les mutuelles chargées de la gestion du régime obligatoire sont couverts dans le cadre d'un contrat collectif puisque les cotisations sont généralement prélevées à la source, alors que juridiquement il s'agit de contrats individuels. 


\section{Un taux de couverture par une complémentaire santé globalement important}

Selon l'enquête BdF06, 91,1 \% des ménages étaient couverts par une complémentaire santé en France en 2006 (cf. tableau 1). Sous l'hypothèse que toute personne appartenant à un ménage couvert est elle-même couverte par une complémentaire santé, 91,6\% des individus seraient donc couverts. On peut alors remarquer que, en dépit du risque de surestimation, ce résultat est très cohérent avec les études réalisées sur l'enquête décennale Santé en 2003 (Marical et Saint Pol, 2007) et l'enquête Santé Protection Sociale (ESPS) de 2006 (Arnould et Vidal, 2008 ; Kambia-Chopin et al., 2008a), qui établissaient ce taux à respectivement $90 \%$ et $93 \%$ de la population.

Si une partie importante de la population française est donc aujourd'hui couverte par une complémentaire santé, la nature même de cette complémentaire varie fortement (cf. encadré 1 pour la définition des types de couverture). Ainsi, dans 21,2\% des ménages interrogés, une personne au moins a déclaré qu'un prélèvement à la source avait été effectué par l'employeur au cours du dernier mois pour une complémentaire santé alors que dans $60 \%$ des cas, seule une dépense auprès d'une mutuelle ou d'une assurance privée a été déclarée au cours des douze derniers mois ${ }^{3}$. En considérant que le type de couverture constaté dans un ménage bénéficie à l'ensemble des membres du ménage, $55,6 \%$ de la population serait couverte par le biais de contrat individuel et $26 \%$ bénéficierait d'un contrat collectif obtenu par le biais de l'employeur de l'un au moins des membres du ménage. Ces résultats sont là encore assez cohérents avec ceux obtenus à partir de l'enquête ESPS 2006, selon lesquels $52 \%$ de la population serait couverte par un contrat individuel et $34 \%$ par un contrat collectif (Arnould et Vidal, 2008).

Enfin, $10 \%$ des ménages bénéficierait gratuitement d'une couverture au titre de la couverture

3. Dans notre échantillon, parmi les 2392 ménages ayant déclaré un prélèvement à la source par l'employeur par au moins l'un des membres du ménages, 294 n'ont déclaré aucune dépense directe de complémentaire santé en complément.
Encadré 1 (suite)

Les personnes couvertes par une complémentaire santé à titre gratuit par l'intermédiaire de la Couverture maladie universelle complémentaire (CMU-C) ne sont pas directement repérables dans l'enquête BdF06, celle-ci ne recueillant pas le statut vis-à-vis de ce dispositif. Le recours à la $\mathrm{CMU}-\mathrm{C}$ a donc été approximé à partir de l'éligibilité estimée des ménages au vu de leurs ressources déclarées. Nous considérons ici qu'un ménage bénéficie de la CMU-C s'il correspond à un foyer éligible au regard des critères en vigueur en 2006 définis par l'assurance maladie selon la loi du 27 juillet 1999. Sont éligibles à la CMUC les individus qui résident en France de façon stable et régulière et qui ont des ressources inférieures à un plafond qui varie selon la composition du foyer du demandeur. Ce plafond s'élevait en 2006 à 598,23 euros par mois pour une personne seule résidant en France métropolitaine. Le foyer CMU comporte le demandeur et son conjoint, son concubin ou son partenaire lié par un PACS, ainsi que les enfants et autres personnes de moins de 25 ans vivant sous le même toit ou figurant sur sa déclaration fiscale ou recevant une pension alimentaire faisant l'objet d'une déduction fiscale. La diversité du recueil des ressources dans l'enquête BDF06 permet ainsi d'approcher de manière très fiable le calcul effectivement effectué par les caisses primaires d'assurance maladie pour définir l'éligibilité à la CMU-C. Néanmoins, cette approximation tend bien évidemment à surestimer le nombre de bénéficiaires de la CMU-C, étant donné l'existence de non recours à ce droit dans la population éligible. Dufour-Kippelen et al. (2006) ont estimé le non recours à la CMUC à $15 \%$.
Les autres ménages non éligibles à la CMU-C et n'ayant déclaré aucune dépense engagée par eux-mêmes et aucun prélèvement effectué par l'employeur sont en revanche considérés comme non couverts. Cette définition tend alors à surestimer le nombre de ménages non couverts puisque sont considérés ici comme non couverts les ménages couverts par le biais de contrats collectifs financés en totalité par l'employeur sans participation des salariés, que l'on sait proposés par $16 \%$ des $43 \%$ d'entreprises proposant une complémentaire d'entreprise (Guillaume et Rochereau, 2010).

Au final, sont donc considérés comme couverts par une complémentaire santé les ménages couverts à titre onéreux ou au titre de la CMU-C. L'enquête Bdf06 ne permet pas de repérer l'existence d'une couverture complémentaire à l'échelle individuelle puisque les dépenses de couverture complémentaire effectuées par les ménages eux-mêmes ou engagées par les employeurs sont repérées à l'échelle du ménage sans autre information sur les bénéficiaires des contrats. L'analyse des déterminants de la couverture complémentaire sera donc réalisée à l'échelle des ménages. Toutefois, pour comparer les taux de couverture obtenus par cette enquête aux taux de couverture obtenus par d'autres sources de données, qui sont le plus souvent calculés au niveau individuel, nous supposerons que toute personne appartenant à un ménage couvert est couverte par une complémentaire santé. Cette hypothèse induira là-encore un biais de surestimation du taux de couverture. 
maladie universelle, dans une approximation de ces ménages par les ménages éligibles à ce dispositif au regard de leurs ressources (cf. encadré 1 pour le détail des critères d'éligibilité à la CMU-C). Ce taux souffre naturellement d'un biais de surestimation puisqu'il repose sur l'hypothèse forte que l'ensemble des ménages éligibles recourt effectivement à ce dispositif alors même que l'existence d'un non recours à la CMU-C a été estimé à environ $15 \%$ (Dufour-Kippelen et al., 2006). De manière attendue, la proportion de ménages éligibles à la CMU-C dans cette enquête est supérieure à la proportion connue de personnes bénéficiaires de ce dispositif selon les autres sources de données : au 31 décembre 2005, 4,7 millions de personnes bénéficiaient de la CMU-C (Boisguérin, 2007), soit $7 \%$ de la population française selon l'enquête ESPS 2006 (Arnould et Vidal, 2008 ; Kambia-Chopin et al., 2008a).

C'est la raison pour laquelle l'analyse conduite par la suite sera réalisée à l'exclusion de la population éligible à la CMU-C.

\section{Un taux de couverture croissant avec le niveau de vie}

Si l'on restreint l'analyse aux 9308 ménages non éligibles à la CMU-C, le taux de couverture pondéré s'élève à 90,2 \% (cf. tableau 1 , colonne 4). Cependant, le taux de couverture et le type de couverture varient fortement selon le niveau de vie des ménages (cf. graphique I ; voir encadré 4 pour une définition du niveau de vie).

\section{Tableau 1}

\section{Types de couverture par une complémentaire santé}

\begin{tabular}{|c|c|c|c|c|c|c|}
\hline \multirow[b]{2}{*}{$\begin{array}{l}\text { Types de couverture } \\
\text { complémentaire santé }\end{array}$} & \multicolumn{3}{|c|}{ Ménages } & \multicolumn{3}{|c|}{ Individus } \\
\hline & $\begin{array}{l}\text { Effectifs non } \\
\text { pondérés }\end{array}$ & $\begin{array}{l}\% \text { dans la } \\
\text { population } \\
\text { totale }\end{array}$ & $\begin{array}{c}\% \text { dans la } \\
\text { population } \\
\text { non éligible } \\
\text { à la CMU-C }\end{array}$ & $\begin{array}{l}\text { Effectifs } \\
\text { non } \\
\text { pondérés }\end{array}$ & $\begin{array}{l}\% \text { dans la } \\
\text { population } \\
\text { totale }\end{array}$ & $\begin{array}{c}\% \text { dans la } \\
\text { population } \\
\text { non éligible } \\
\text { à la CMU-C }\end{array}$ \\
\hline Pas de couverture & 873 & 8,5 & 9,4 & 2045 & 8,1 & 8,8 \\
\hline $\begin{array}{l}\text { Couverture par une complémentaire } \\
\text { santé dont }\end{array}$ & 9363 & 91,5 & & 23315 & 91,9 & \\
\hline $\begin{array}{l}\text { Couverture à totre } \\
\text { gratuit (CMU-C) }\end{array}$ & 928 & 9,1 & & 2223 & 8,8 & \\
\hline $\begin{array}{l}\text { Couverture à titre } \\
\text { onéreux dont }\end{array}$ & 8435 & 82,4 & 90,6 & 21092 & 83,2 & 91,2 \\
\hline $\begin{array}{l}\text { Au moins par } \\
\text { un contrat collectif } \\
\text { fourni par l'employeur }\end{array}$ & 2392 & 23,3 & 25,7 & 7071 & 27,9 & 30,6 \\
\hline $\begin{array}{l}\text { Par contrat individuel } \\
\text { uniquement }\end{array}$ & 6043 & 59,0 & 64,9 & 14021 & 55,3 & 60,6 \\
\hline Échantillon de référence & & 10236 & 9308 & & 25360 & 23137 \\
\hline
\end{tabular}

\begin{tabular}{|l|l|c|c|c|c|c|}
\hline \multicolumn{1}{|c|}{ Lecture en \% pondéré } & & \% pondéré & \% pondéré & & \% pondéré & \% pondéré \\
\hline Pas de couverture & & 8,9 & 9,8 & & 8,4 & 9,4 \\
\hline $\begin{array}{l}\text { Couverture par une } \\
\text { complémentaire santé dont }\end{array}$ & 91,1 & & & 91,6 & \\
\hline $\begin{array}{c}\text { Couverture à totre } \\
\text { gratuit (CMU-C) }\end{array}$ & 9,9 & & & 9,8 & \\
\hline Couverture à titre onéreux dont & & 81,2 & 90,2 & & 81,7 & 90,6 \\
\hline $\begin{array}{l}\text { Au moins par } \\
\text { un contrat collectif } \\
\text { fourni par l'employeur }\end{array}$ & 21,2 & 23,6 & & 26,1 & 28,9 \\
\hline $\begin{array}{l}\text { Par contrat individuel } \\
\text { uniquement }\end{array}$ & & 59,9 & 66,6 & & 55,6 & 61,7 \\
\hline
\end{tabular}

Lecture : Parmi les 10236 ménages interrogés, 9363 sont couverts par une complémentaire santé, soit un taux de couverture de $91,5 \%$ Parmi ceux-ci, les 928 ménages éligibles à la CMU-C sont considérés comme couverts à titre gratuit. Parmi les 9308 ménages non éligibles à la CMU-C, 8435 ménages sont couverts à titre onéreux, soit un taux de 90,6\%.

Les pourcentages sont aussi calculés en tenant compte de la variable de pondération associée à chaque ménage disponible dans la base de données.

Champ : Ménages enquêtés en France métropolitaine dont la personne de référence est âgée de 18 ans et plus (10 236 ménages). Les pourcentages sont calculés en tenant compte des pondérations.

Source : Enquête Budget de Famille 2006. 
Ainsi, parmi la population des ménages non éligibles à la CMU-C, $19 \%$ des ménages appartenant au $10 \%$ de ménages ayant le niveau de vie le plus faible (ayant donc un niveau de vie inférieur à 827 euros par unité de consommation) ne disposent d'aucune complémentaire santé alors que le taux de non couverture n'excède pas $10 \%$ à partir du $4^{\mathrm{e}}$ décile. Ce résultat confirme là-encore les résultats des études précédentes fondés sur l'enquête Santé 2003 et l'enquête ESPS 2006, qui avaient souligné le rôle déterminant du revenu dans l'accès à la complémentaire santé (Arnould et Vidal, 2008 ; Marical et de Saint Pol, 2007 ; Kambia-Chopin et al., 2008a).

Le type de couverture complémentaire varie également selon le niveau de vie des ménages : la proportion de ménages bénéficiant d'un contrat collectif augmente avec le niveau de vie, de manière cohérente avec les études précédentes ayant montré que les contrats collectifs bénéficiaient plus fréquemment aux cadres des grandes entreprises (Kambia-Chopin et al., 2008a ; Guillaume et Rochereau, 2010).

Cette tendance est confirmée par l'analyse du niveau de vie moyen des ménages selon leur type de couverture. Parmi les ménages non éligibles à la CMU-C, le niveau de vie mensuel moyen était de 1392 euros pour les ménages non couverts, de 1539 euros pour les ménages couverts individuellement et de 1733 euros pour les ménages bénéficiant d'un contrat collectif (avec des écarts-types respectifs de 1058 , 944 et 757).

\section{Des dépenses de complémentaire santé croissantes avec le niveau de vie}

Les informations recueillies dans l'enquête BdF06 sur le montant des dépenses engagées par les ménages eux-mêmes ainsi que sur la somme des prélèvements effectués par les employeurs des différents membres du ménage pour l'achat d'un contrat de couverture complémentaire permettent d'étudier finement les dépenses directes et indirectes des ménages pour l'achat de contrats d'assurance complémentaire. L'analyse est donc à présent restreinte aux 8435 ménages couverts par une complémentaire santé à titre onéreux, que ce soit dans le cadre d'un contrat individuel ou d'un contrat collectif.

Le montant moyen des dépenses de complémentaire santé par tête et par an s'élève à 579 euros dans l'ensemble des ménages couverts à titre

\section{Graphique I}

Types de complémentaire santé selon le niveau de vie mensuel des ménages en euros

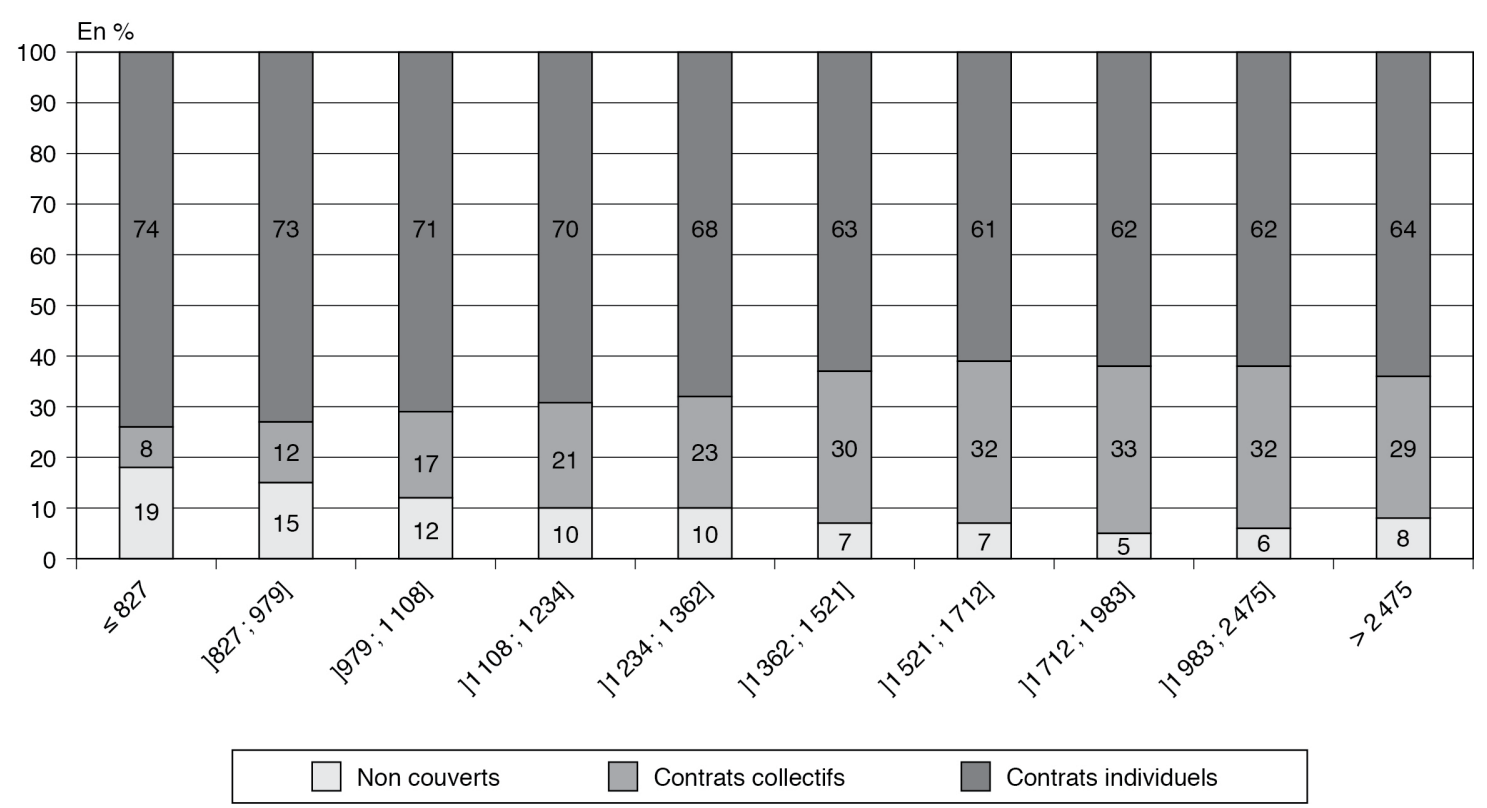

Lecture : Parmi les $10 \%$ des ménages ayant le niveau de vie le plus faible au sein des ménages non éligibles à la CMU-C, $74 \%$ sont couverts par un/des contrat(s) individuel(s) uniquement, $8 \%$ par au moins un contrat collectif au sein du ménage et $19 \%$ ne sont pas couverts. Les déciles de niveau de vie ont été calculés à partir de la distribution pondérée des revenus disponibles mensuels par unité de consommation de l'ensemble des ménages hors éligibles à la CMU-C ( $n=9$ 308).

Champ : Ménages enquêtés en France métropolitaine dont la personne de référence est âgée de 18 ans et plus et non éligibles à la CMU-C (9 308 ménages). Les calculs prennent en compte les pondérations.

Source : Enquête Budget de Famille 2006. 
onéreux mais varie selon le type de couverture (cf. tableau 2). Ainsi, les dépenses des ménages couverts individuellement s'élèvent à 553 euros en moyenne ; elles sont sensiblement inférieures à celles des ménages bénéficiant au moins d'une couverture d'entreprise qui s'élèvent à 652 euros. On peut tout d'abord remarquer que le montant moyen des dépenses de complémentaire santé des ménages couverts à titre individuel estimé à partir de données de l'enquête BdF06 est très proche de l'estimation réalisée par Grignon et Kambia-Chopin (2009 ; 2010), à partir des données de l'enquête ESPS 2004, qui s'élevait à 527 euros. L'estimation des dépenses de complémentaire des ménages couverts par le biais de l'employeur ne peut, quant à elle, être confrontée à aucune source, faute de données sur les prélèvements à la source effectués par les employeurs dans les autres enquêtes ménages. Le montant plus élevé des dépenses de complémentaire des ménages bénéficiant d'une couverture d'entreprise peut en outre paraître surprenant, dans la mesure où ces ménages bénéficient d'une participation de l'employeur, qui s'élève en moyenne à $57 \%$ du coût des contrats (Guillaume et Rochereau, 2011). Cependant, ceci s'explique d'une part par la qualité supérieure des contrats de couverture d'entreprise (Guillaume et Rochereau, 2011) et, d'autre part, par le fait que les ménages sont très souvent couverts par plusieurs contrats, combinant notamment couverture individuelle et couverture collective (cf. encadré 2 pour une discussion sur la crédibilité des dépenses déclarées dans Bdf06).

Le montant des dépenses de complémentaire santé des ménages varie sensiblement avec leur niveau de vie (cf. graphique II). Les dépenses moyennes des ménages couverts à titre individuel uniquement restent inférieures à 565 euros dans les huit premiers déciles (les $80 \%$ les plus pauvres), sans que l'on puisse véritablement définir une tendance nette, mais augmentent très fortement parmi les $20 \%$ des ménages les plus riches. Elles sont égales à 615 euros dans le neuvième décile et 728 euros dans le dixième.

Bien que les dépenses des ménages bénéficiant d'une couverture d'entreprise soient supérieures à celles des ménages couverts à titre individuel à partir du quatrième décile de niveau de vie, leur montant reste assez comparable dans les sept premiers déciles et inférieur à 565 euros. Elles augmentent ensuite fortement dans les trois derniers déciles de niveau de vie pour atteindre plus de 1000 euros parmi les $10 \%$ les plus riches.

Comme nous ne disposons pas d'informations précises sur les contrats, il n'est pas possible de savoir si les dépenses supérieures correspondent à des contrats de meilleure qualité. Néanmoins, l'étude d'Arnould et Vidal (2008) montre que la nature et le montant des prestations prises en charge augmentent avec le prix, en particulier dans la cas des contrats individuels.

\section{Accessibilité financière à la complémentaire santé}

L'analyse de la distribution des dépenses de complémentaire santé selon le niveau de vie conduit à s'interroger sur l'effort consenti par les ménages pour la souscription d'un contrat de complémentaire santé. Le taux d'effort représente la part du revenu disponible consacré aux dépenses de complémentaire santé. Il peut être calculé pour les ménages couverts individuellement ou pour ceux couverts par le biais de l'employeur. Dans le premier cas, il

Tableau 2

Dépenses de complémentaire santé par tête et par an (en euros) selon les types de couverture

\begin{tabular}{|c|c|c|c|}
\hline \multirow[t]{2}{*}{ Types de couverture } & \multirow[t]{2}{*}{ Effectifs } & $\begin{array}{c}\text { Niveau moyen } \\
\text { des dépenses de } \\
\text { complémentaire santé par } \\
\text { tête et par an (en euros) }\end{array}$ & \multirow[t]{2}{*}{ Écart-type } \\
\hline & & Par tête & \\
\hline Ménages couverts individuellement & 6043 & 553 & 401 \\
\hline Ménages couverts par le biais de l'employeur & 2392 & 652 & 664 \\
\hline Ensemble des ménages couverts à titre onéreux & 8435 & 579 & 492 \\
\hline
\end{tabular}

Lecture : Parmi l'ensemble des ménages couverts à titre onéreux, le niveau moyen des dépenses de complémentaire santé engagées directement par les ménages ou indirectement par le biais de prélèvements à la source effectués par les employeurs s'élève 579 euros par tête et par an.

Champ : Ménages enquêtés en France métropolitaine dont la personne de référence est âgée de 18 ans et plus, non éligibles à la CMU-C et couverts par une complémentaire santé à titre onéreux (8 435 ménages, dont 6043 couverts à titre individuel et 2392 couverts par l'employeur). Les calculs prennent en compte les pondérations.

Source : Enquête Budget de Famille 2006. 
correspond à la part des dépenses de complémentaire santé dans le revenu disponible total des ménages. Dans le second cas, il correspond à la part de l'ensemble des dépenses de complémentaire santé, soit par le biais de prélèvement de l'employeur, soit engagées directement par les ménages auprès d'organismes complémentaires, dans le revenu disponible total des ménages augmenté des prélèvements effectués par l'employeur, qui correspondent à une rémunération en nature.

En moyenne, les ménages couverts par le biais de contrats individuels consacrent $4,5 \%$ de leur revenu disponible pour acquérir une complémentaire santé. Là encore, le taux d'effort varie en fonction du revenu disponible des ménages, passant de 8,5\% en moyenne pour les $10 \%$ des ménages les plus pauvres à $2,3 \%$ pour les $10 \%$ des ménages les plus riches (cf. graphique III). Les ménages bénéficiant d'une couverture par le biais de leur employeur consacrent, quant à eux, en moyenne $5 \%$ de leur revenu disponible à leurs dépenses de complémentaire santé, leur taux d'effort allant de $10,7 \%$ parmi les $10 \%$ des ménages les plus pauvres à 3,3\% parmi les $10 \%$ des ménages les plus riches. La différence entre les taux d'effort relatif aux contrats collectifs et aux contrats individuels s'explique sans doute par des différences de qualité. En effet, les contrats collectifs offrent en moyenne des garanties plus élevées et sont donc plus onéreux (Arnould et Vidal, 2008 ; Couffinhal et al., 2004), même si, du fait des d'économies d'échelle et du pouvoir de négociation des entreprises, le coût des contrats collectifs est moins élevé que celui des contrats individuels à qualité donnée (Perronnin et al., 2011).

Au regard des taux d'effort observés pour les ménages appartenant aux premiers déciles de revenus, il est naturel de s'interroger sur l'accessibilité financière à la couverture

\section{Graphique II \\ Niveau de dépenses de complémentaire santé par tête et par an selon le type de couverture et le niveau de vie mensuel des ménages}

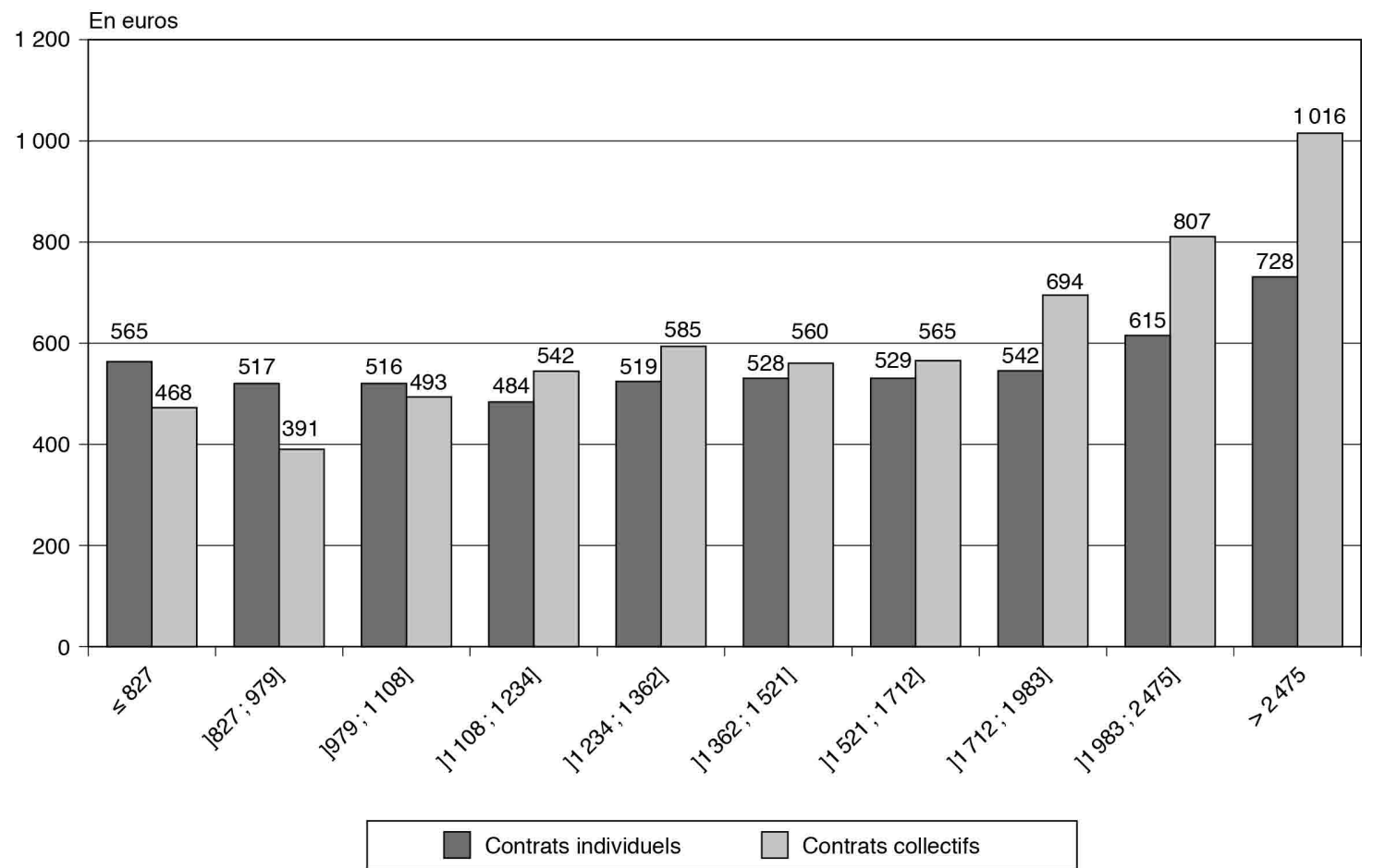

Lecture : Parmi les $10 \%$ des ménages ayant le niveau de vie le plus faible au sein des ménages non éligibles à la CMU-C, la dépense moyenne pour la complémentaire santé est de 565 euros par tête par an pour les ménages couverts par contrat(s) individuel(s) uniquement et de 468 euros pour les ménages couverts au moins par un contrat collectif.

Champ : Ménages enquêtés en France métropolitaine dont la personne de référence est âgée de 18 ans et plus et non éligibles à la CMU-C et couverts par une complémentaire santé à titre onéreux (8 435 ménages, dont 6043 couverts à titre individuel et 2392 couverts par l'employeur). Les calculs prennent en compte les pondérations.

Source : Enquête Budget de Famille 2006. 
complémentaire santé en France. Compte tenu de la liberté de choix laissé à chacun de souscrire ou non une assurance, rien n'assure qu'un individu pleinement informé ne choisisse pas, en toute rationalité, de ne pas souscrire une assurance complémentaire santé, compte tenu de faibles dépenses de santé anticipées ou de préférences particulières, telles qu'une faible aversion au risque, une faible préférence pour la santé ou une forte préférence pour le

Encadré 2

\section{CADRAGE MACROÉCONOMIQUE SUR LA CRÉDIBILITÉ DES DÉPENSES DE COMPLÉMENTAIRE SANTÉ DÉCLARÉES DANS BDF06}

Les dépenses de complémentaire santé étant déclarées par les ménages dans l'enquête BdF06, il convient de s'interroger sur leur crédibilité.

Selon nos résultats le niveau des dépenses par tête et par an s'élève alors à 579 euros en moyenne pour les ménages couverts à titre onéreux, à 553 euros pour les ménages couverts uniquement à titre individuel et à 652 euros pour les ménages bénéficiant au moins d'une couverture d'entreprise.

Ces montants peuvent paraître élevés au regard des montants de cotisations des contrats les plus souvent souscrits. En effet, selon l'enquête 2006 de la Drees auprès des mutuelles, des institutions de prévoyance et des sociétés d'assurance sur les trois contrats individuels et des deux contrats collectifs les plus fréquemment souscrits auprès de chaque organisme, les cotisations moyennes par bénéficiaire s'établissent à 463 euros par an pour les contrats individuels et à 421 euros pour les contrats collectifs (Arnould et Vidal, 2008). Toutefois, ces deux informations ne sont pas directement comparables. L'enquête de la DREES n'est pas représentative de l'ensemble des contrats et renseigne sur le prix de ces contrats modaux, alors que nos résultats portent sur les dépenses totales engagées par les ménages pour leur couverture complémentaire. Or les ménages sont très souvent couverts par plusieurs contrats, combinant notamment couverture individuelle et couverture collective. Dans notre échantillon, parmi les 2392 ménages ayant déclaré un prélèvement à la source par l'employeur par au moins l'un des membres du ménages, seuls 294 n'ont déclaré aucune dépense directe de complémentaire santé en complément.

Il est également légitime de s'interroger sur la crédibilité des montants déclarés au regard des dépenses de santé effectivement financées par les organismes complémentaires.

Selon les comptes de la santé, la Consommation de soins et biens médicaux (CSBM) s'est élevée en 2006 à 156,6 milliards d'euros, soit 8,7 \% du Produit intérieur brut (Fenina et Geoffroy, 2007). Les dépenses prises en charge par les organismes complémentaires se sont élevées à 20,4 milliards d'euros, soit $13 \%$ de la CSBM $(7,4 \%$ par les mutuelles, 3,2 \% par les assurances et 2,4 \% par les instituts de prévoyance).

En 2006, il y avait en France environ 26,7 millions de ménages, composés en moyenne de 2,3 personnes
(Insee, 2010). Sous l'hypothèse que $81,2 \%$ des ménages sont couverts à titre onéreux et qu'ils ont eu en moyenne une dépense de complémentaire santé égale à 579 euros par personne, les dépenses totales des ménages en complémentaire santé s'élèvent à :

Estimation des dépenses totales des ménages en complémentaire santé

$$
=579 \times 0,812 \times 26,7 \times 2,3=28,9 \text { milliards }
$$

À ces dépenses engagées par les ménages s'ajoutent les dépenses engagées par les employeurs. Sous I'hypothèse que $21,2 \%$ des ménages bénéficient d'un contrat collectif, que les employeurs prennent en moyenne $57 \%$ du coût des contrats (Guillaume et Rochereau, 2011) et que le prix moyen des contrats financés par les employeurs s'établit au prix modal de 421 euros, les dépenses de complémentaire santé des entreprises s'établissent à :

Estimation des dépenses totales des employeurs en complémentaire santé

$$
=421 \times 0,57 \times 0,212 \times 26,7 \times 2,3=3,1 \text { milliards }
$$

Le montant total des dépenses de complémentaires engagées par les ménages et les entreprises s'élèverait à 32 milliards. Ce montant est à mettre en regard des dépenses de santé remboursées par les organismes de complémentaire de 20,4 milliards. En assimilant le montant des dépenses de complémentaires ainsi estimé au montant des primes collectées par les organismes de complémentaire santé (OCAM, organisme de complémentaire d'assurance maladie), le ratio remboursements/primes des OCAM s'élèverait donc à $64 \%(=20,4 / 32)$.

Dans le rapport 2009 de la Drees " La situation financière des organismes assurant une couverture santé ", ce ratio est évalué en 2007 (nous ne disposons pas des mêmes évaluations pour 2006) à $70 \%$ pour les assureurs, $77 \%$ pour les instituts de prévoyance et $80 \%$ pour les mutuelles. Au prorata de leur importance relative dans les consommations de soins (compte de la santé 2007) on peut estimer le ratio remboursements/primes moyen des OCAM à $77 \%$. Ceci suggérait que les dépenses de complémentaires santé sont surestimées dans l'enquête à hauteur de 5,5 milliards, soit $17 \%$ du montant de ces dépenses, ce qui doit naturellement être pris considération dans l'interprétation de nos résultats. 
présent ${ }^{4}$. Il est donc, dans ce cadre, plus cohérent pour le législateur de viser l'accessibilité à la complémentaire santé, c'est-à-dire la possibilité financière qu'un ménage a d'acheter un contrat, plutôt que de rechercher la couverture universelle de toute la population ${ }^{5}$. Bundorf et Pauly (2006) discutent de cette notion et distinguent une définition normative de l'accessibilité d'une définition positive (cf. encadré 3). Nous retiendrons l'acception normative qui repose sur la définition a priori d'un seuil de pauvreté auquel sera comparé le revenu disponible des ménages diminué du montant nécessaire à l'achat d'une complémentaire santé.

De manière usuelle, nous avons retenu un seuil de pauvreté égal à $60 \%$ du revenu disponible par unité de consommation médian, calculé à partir de la distribution des revenus renseignés dans l'enquête BdF06. Ce seuil s'établit à 773 euros mensuel par unité de consommation et conduit à considérer que $15,0 \%$ des ménages sont pauvres ${ }^{6}$. La plupart des ménages « pauvres » au regard de cet indicateur s'avèrent être éligibles à la CMU-C. Toutefois, 6,8 \% de la population des ménages non éligibles à la CMU-C ont encore un niveau de vie inférieur au seuil de pauvreté.

Une analyse plus fine ne portant cette fois-ci que sur les ménages pauvres et non éligibles à la CMU-C, c'est-à-dire sur les ménages devant financer eux-mêmes leur complémentaire santé alors qu'ils disposent de ressources extrêmement modestes, montre que $18,7 \%$ de ces ménages ne sont pas couverts par une complémentaire santé, alors que la proportion de ménages dépourvus de complémentaire tombe à $9,2 \%$ au sein des ménages dont les revenus sont supérieurs au seuil de pauvreté.

En reprenant la définition de Bundorf et Pauly (2006), il est possible de calculer un taux de

4. Théoriquement, plus une personne est averse au risque, plus elle cherche à s'assurer pour se prémunir contre des variations aléatoires de son revenu induites par les dépenses de santé. Barsky et al. (1997) et Monheit et Vistnes (2006) montrent ainsi sur données américaines que les individus les moins averses au risque sont plus souvent non couverts et Doiron et al. (2008) montrent sur données australiennes que les individus qui adoptent des comportements à risque sont plus souvent non assurés. Le recours à l'assurance complémentaire peut également être vu comme un préengagement à recourir au système de soins (Grignon et Kambia-Chopin, 2009) et serait assimilable à un investissement en capital santé. Si l'on se réfère au cadre théorique du modèle de capital santé (Grossman, 2000), la demande d'assurance devrait donc être d'autant plus forte chez les personnes ayant une forte préférence pour la santé et une faible préférence pour le présent, et donc parmi les plus riches et les éduqués.

5. Voir Jusot et Wittwer (2009) pour une discussion sur les hésitations et incohérences du législateur sur ce point.

6. Le seuil de pauvreté calculé à partir des données de BdF06 est sensiblement inférieur au seuil de pauvreté calculé par l'Insee sur la base de revenus fiscaux qui s'établissait à 880 euros pour l'année 2006 (Goutard, Pujol, 2008) ce qui laisse supposer une sous-déclaration des revenus dans BdF06. Toutefois, il conduit à une estimation proche du taux de pauvreté, puisque, selon les données fiscales, 13,2\% des personnes vivaient en dessous $d u$ seuil de pauvreté en 2006.

\section{Graphique III \\ Taux d'effort en complémentaire santé selon le type de couverture et le niveau de vie mensuel des ménages}

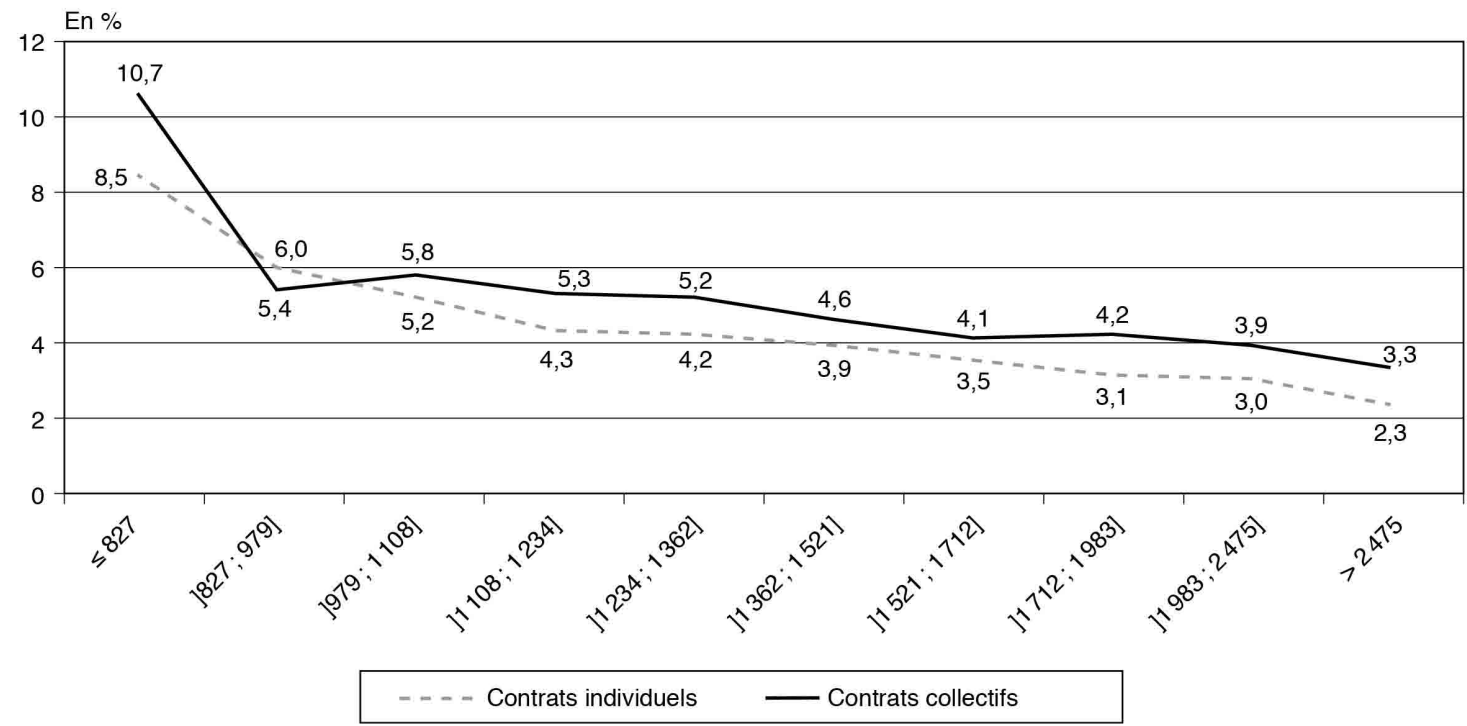

Lecture : Parmi les $10 \%$ des ménages ayant le niveau de vie le plus faible au sein des ménages non éligibles à la CMU-C, le taux d'effort moyen pour l'achat d'une complémentaire santé est de $8,5 \%$ pour les ménages couverts par contrat(s) individuel(s) uniquement et de $10,7 \%$ pour les ménages couverts au moins par un contrat collectif.

Champ : Ménages enquêtés en France métropolitaine dont la personne de référence est âgée de 18 ans et plus et non éligibles à la CMU-C et couverts par une complémentaire santé à titre onéreux (8 435 ménages, dont 6043 couverts à titre individuel et 2392 couverts par l'employeur).

Source : Enquête Budget de Famille 2006. 
pauvreté « net » correspondant au pourcentage de ménages ayant un revenu ajusté des dépenses de complémentaire santé inférieur au seuil de pauvreté. En l'absence de norme sur un contrat de complémentaire santé que l'on pourrait considérer comme un contrat minimum (la CMUC-C étant gratuite), nous avons retenu le niveau moyen des dépenses de complémentaire santé souscrite à titre individuel par tête et par an comme approximation du montant nécessaire à l'achat d'un contrat de complémentaire santé de qualité moyenne sur le marché ${ }^{7}$. Le taux de pauvreté net est donc égal à : (Revenu total - 553*nombre de personnes)/nombre d'unité de consommation $<773$.

Le taux de ménages pour lesquels la complémentaire santé peut être considérée comme non accessible est de 18,5\% dans l'ensemble de la population et encore de $10,3 \%$ dans la population des ménages non éligibles à la CMU-C. Il vient donc que dans chacune des deux souspopulations, l'achat d'une complémentaire santé conduirait 3,5\% des ménages à tomber sous le seuil de pauvreté (cf. graphique IV).

Pour savoir dans quelle mesure l'aide complémentaire santé pouvait améliorer l'accessibilité financière à la complémentaire pour les ménages modestes, l'analyse précédente a été reconduite en ajoutant le montant de cette subvention à l'ensemble des ménages éligibles à cette aide au regard de leur revenu et des seuils en vigueur au moment de l'enquête. Cette subvention était égale en 2006 égale à 100 euros par personne âgée de moins de 25 ans, 200 euros pour les personnes âgées de 26 à 59 ans et 400 euros pour les personnes âgées de 60 ans et plus ${ }^{8}$. L'ACS (Aide complémentaire santé) modifie peu l'accessibilité financière à la complémentaire : la complémentaire reste inaccessible à $18,2 \%$ de l'ensemble des ménages et à $9,9 \%$ dans la population des ménages non éligibles à la CMU-C.

\section{Les déterminants de la demande de complémentaire santé en 2006}

Afin d'approfondir cette analyse de l'accessibilité financière à la complémentaire santé, une analyse économétrique a été menée afin d'étudier, au sein de la population non couverte par la CMU-C et non couverte par le biais de l'employeur, les

7. En l'absence d'information sur la qualité des contrats, il n'est pas possible d'étudier l'éventuelle dégressivité des primes avec le nombre de personnes couvertes. L'absence de prise en considération de cette éventuelle dégressivité ne conduit à aucune surestimation globale des primes mais peut induire une surestimation du coût de la complémentaire pour les ménages avec enfants et une sous-estimation pour les ménages sans enfant. Cette déformation peut alors conduire à une surestimation du taux de pauvreté net si les ménages avec enfants sont les plus fragiles face à la pauvreté.

8. Les montants sont aujourd'hui de 100 euros par personne de moins de 16 ans, de 200 euros par personne de 16 à 49 ans, de 350 euros par personne de 50 à 59 ans et de 500 euros par personne de 60 ans et plus. En outre, le plafond de l'ACS est depuis

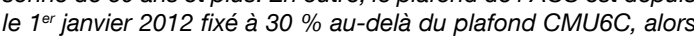
qu'il était égal à $20 \%$ du plafond CMU-C en 2006. L'accessibilité financière de la complémentaire est donc aujourd'hui légèrement améliorée. Notons cependant que le taux de d'utilisation du chèque santé demeure faible (voir Guthmuller et al., 2012).

Encadré 3

\section{L'ACCESSIBILITÉ FINANCIÈRE AU SENS DE BUNDORF ET PAULY}

Bundorf et Pauly (2006) donnent deux définitions de l'accessibilité financière à une assurance santé.

La première définition s'appuie sur la définition a priori d'un revenu jugé minimal pour subvenir aux besoins essentiels d'un ménage donné, $\bar{G}$, et d'un niveau de dépenses en assurance santé jugé minimal, $\bar{x}$, pour ce même ménage. L'assurance santé sera jugée financièrement accessible pour ce ménage si : $y-\bar{x} \overline{\underline{G}}$, où $\boldsymbol{y}$ désigne le revenu disponible du ménage considéré. Les auteurs parlent de définition normative de l'accessibilité financière. Ce type de définition est par exemple utilisé pour juger de l'accessibilité financière au logement. Elle suppose de définir le niveau de revenu minimal $\bar{G}$ à partir de la valeur marchande d'un panier de biens jugé nécessaire, c'est-à-dire de recourir à une mesure absolue de la pauvreté. Ce type d'indicateur de la pauvreté n'étant pas couramment estimé en France nous élargissons l'application de cette définition à une mesure relative de la pauvreté.
La seconde définition de l'accessibilité financière s'appuie sur le comportement observé des ménages. En deux mots, on dira qu'un ménage a accès à l'assurance santé si la majorité des ménages comparables sont couverts par une assurance santé. Pratiquement, cette mesure de l'accessibilité financière est concrétisée à l'aide d'une régression logistique de la probabilité de détenir une assurance santé en contrôlant du revenu et des facteurs susceptibles d'influencer le prix de la couverture (les variables influençant le risque santé : âge, taille du ménage, lieu de résidence...). Les résidus de cette régression sont alors censés refléter les préférences des ménages. En calculant les probabilités simulées, il est alors possible de dire, pour chaque jeu des variables explicatives, si l'assurance santé est accessible ou non, en fixant arbitrairement un seuil (50\% par exemple). La difficulté de cette méthode tient essentiellement à l'identification de variables explicatives influençant exclusivement le risque santé. 
déterminants de la décision de souscrire un contrat de complémentaire santé et du choix des dépenses engagées pour cette couverture. La modélisation a été réalisée à l'aide d'un modèle Tobit généralisé afin d'étudier conjointement les déterminants de ces deux décisions, tout en autorisant ces derniers à être différents. Nous avons alors utilisé le sexe de la personne de référence du ménage comme variable d'exclusion puisque cette variable était associée à la probabilité d'être couvert - les femmes ayant une plus grande propension à être couvertes - mais pas au montant des dépenses de santé. L'analyse confirme alors la pertinence du modèle retenu dans la mesure où le ratio de Mills est significatif dans la deuxième équation, ce qui indique une corrélation significative entre les résidus des deux équations (pour la méthode, cf. encadré 4).

Cette analyse toutes choses égales par ailleurs confirme alors le rôle important joué par le revenu tant sur la probabilité d'être couvert que sur le montant des dépenses engagées, en cohérence avec les résultats de Saliba et Ventelou (2007) et Grignon et Kambia-Chopin (2009) établis sur les données des enquêtes ESPS. Toutefois, la forme de la relation entre le revenu et la demande de complémentaire diffère selon les étapes de la décision. Alors que la probabilité d'être couvert individuellement croît continûment avec le niveau de revenu, seule la perception de hauts revenus semble influencer significativement le montant des dépenses de complémentaire.

L'analyse confirme ensuite l'existence de fortes inégalités sociales dans la détention et dans la qualité de la couverture complémentaire (cf. tableau 3). La possession d'une couverture complémentaire est ainsi liée à la situation de la personne de référence sur le marché du travail. Les étudiants et les chômeurs ont ainsi une probabilité plus faible d'être couverts que les personnes occupant un emploi, alors que les retraités ont une probabilité plus forte d'être couverts. Les résultats font également apparaître une relation significative entre le niveau d'instruction et la détention de couverture. Les personnes sans diplôme ont clairement une probabilité plus faible d'être couvertes que les diplômés du premier cycle. Il est cependant intéressant de remarquer que le lien entre diplôme et couverture n'est pas monotone. En effet, si la significativité est moins nette, on note néanmoins que les diplômés de second cycle et du supérieur ont, eux aussi, une probabilité plus faible de détenir une complémentaire santé. Ce résultat, fragile, vient s'ajouter au constat que les cadres se distinguent des professions intermédiaires par une probabilité d'être couverts non significativement différente de celle des ouvriers et des employés. On peut y voir l'expression d'une rationalité aiguisée chez les plus diplômés et les plus qualifiés plus enclins à mettre en œuvre un arbitrage fin entre coût de la prime et risque. Cependant, la moindre probabilité d'engager une dépense de complémentaire à titre individuel des plus diplômés et des cadres pourraient également s'expliquer par le bénéfice plus fréquent d'une couverture à titre gratuit par leurs employeurs. On ne peut également exclure l'existence d'un biais de sélection lié à l'exclusion des personnes couvertes par leur employeur à titre onéreux qui n'a pu être traité faute de variable identifiante satisfaisante dans les données telle

\section{Graphique IV}

Accessibilité au sens financier de la complémentaire santé (CS)

Ensemble de la population des ménages
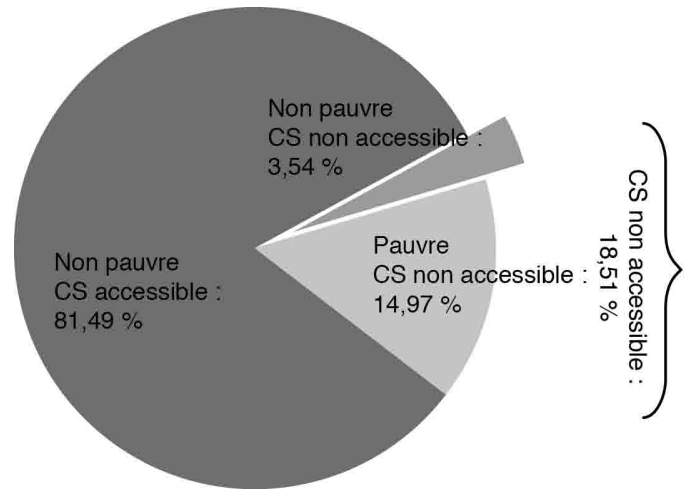

Population des ménages non éligible à la CMU-C

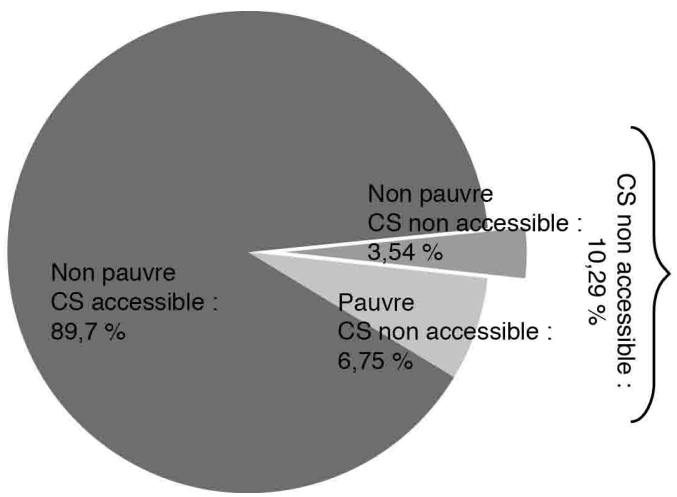

Lecture : Parmi l'ensemble de la population, la complémentaire n'est pas accessible financièrement à 18,51 \% de la population totale, dans la mesure où leur revenu disponible est inférieur au seuil de pauvreté après déduction des dépenses de complémentaire santé. Champ : Ménages enquêtés en France métropolitaine dont la personne de référence est âgée de 18 ans et plus. Les calculs prennent en compte les pondérations.

Source : Enquête Budget de Famille 2006. 
que le secteur d'activité. On note enfin que les artisans et commerçants recourent moins à une assurance complémentaire santé, sans que l'on sache si cela révèle des préférences particulières ou le souci de diminuer les charges pour faire face à une variabilité des revenus propres à ces professions. À l'inverse, les agriculteurs ont, quant à eux, une probabilité plus forte de se couvrir. Ces résultats se retrouvent dans l'étude de Perronnin et al. (2011).

\section{Encadré 4}

\section{ANALYSE DES DÉTERMINANTS DE LA DEMANDE DE COMPLÉMENTAIRE SANTÉ}

Afin d'analyser les déterminants de la demande de complémentaire santé, l'analyse a été réalisée à partir de l'échantillon pour lequel le fait d'être couvert et le montant des dépenses engagées relèvent d'un choix. Nous avons donc exclu les 928 ménages éligibles à la CMU-C ainsi que les 2392 ménages couverts par un contrat collectif fourni par l'employeur de l'un au moins des membres du ménage. On peut en effet se demander si certains ménages repérés comme couverts " multiples " ne sont pas uniquement couverts par le biais de leur employeur car certains déclarent payer directement de leur poche exactement le même montant que la somme qu'ils déclarent prélevée par leur employeur.

Cet échantillon comprend ainsi les 873 ménages non couverts et les 6043 ménages couverts uniquement par un contrat individuel auprès d'une mutuelle ou d'une assurance privée.

L'analyse a été réalisée à l'aide de l'estimation d'un modèle Tobit généralisé qui permet d'étudier d'une part les déterminants du recours à la complémentaire santé et d'autre part les déterminants du montant des dépenses engagées. Ce modèle suppose l'existence de deux variables latentes, $Y_{1 i}^{*}$ la propension à recourir à une complémentaire santé des ménages $i$ et $Y_{2 i}^{*}$ leur demande de complémentaire santé, et d'une variable observable, $Y_{2 i}$ le montant de la dépense effectivement engagée par les ménages $i$, telles que :

$Y_{1 i}^{*}=X_{1 i}^{*} \beta_{1}+u_{1 i}$

$Y_{2 i}^{*}=X_{2 i}^{*} \beta_{2}+u_{2 i}$

avec $Y_{2 i}=Y_{2 i}^{*}$ si $Y_{1 i}^{*}>0$ et $Y_{2 i}=0$ si $Y_{1 i}^{*} \leq 0$

avec $X_{1 i}^{*}$ et $X_{2 i}^{*}$, les vecteurs des variables explicatives, $\beta_{1}$ et $\beta_{2}$ vecteurs des paramètres associés, $u_{1 i}$ et $u_{2 i}$ les erreurs distribuées selon une normale bivariée d'écart type $\sigma_{1}$ et $\sigma_{2}$ et de covariance $\sigma_{12}$.

Ce modèle a été estimé à l'aide d'une procédure de Heckman en deux étapes. La première étape consiste à estimer les paramètres de l'équation (1) à l'aide d'un modèle Probit simple. La seconde étape consiste à estimer à l'aide d'un modèle de régression linéaire les paramètres de l'équation (2) au sein uniquement des personnes ayant effectivement engagées des dépenses $\left(Y_{1 i}^{*}>0\right)$. Cependant, afin de prendre en compte le biais de sélection induit par l'exclusion des ménages non assurés dans cette seconde étape, le ratio de Mills de la première équation est introduit dans la seconde, ce qui revient à estimer l'équation (3) :

$Y_{2 i}^{*}=X_{2 i}^{*} \beta_{2}+\frac{\sigma_{12}}{\sigma_{1}} \frac{\varphi\left(\frac{X_{1 i}^{*} \hat{\beta}_{1}}{\hat{\sigma}_{1}}\right)}{\phi\left(\frac{X_{1 i}^{*} \hat{\beta}_{1}}{\hat{\sigma}_{1}}\right)}+v_{2 i}$

où $\varphi$ et $\Phi$ sont respectivement la densité et la fonction de répartition de la loi normale centrée réduite et $v_{2 i}$ un terme d'erreur distribué selon une loi normale identiquement et indépendamment distribuée.

De manière usuelle, le montant des dépenses de complémentaire santé par tête et par an a été considéré en logarithme dans cette seconde étape, afin de réduire le poids des valeurs extrêmes et limiter les risques d'hétéroscédasticité.

Plusieurs variables ont été introduites dans les vecteurs $X_{1 i}^{*}$ et $X_{2 i}^{*}$, afin d'étudier les déterminants de la demande d'assurance complémentaire santé.

Afin d'obtenir une approximation du niveau de risque du ménage, nous avons retenu un indicateur dichotomique opposant les ménages dont au moins un des membres souffre de limitations fonctionnelles, aux autres ménages. Celui-ci a été construit à l'aide de la question suivante renseignée pour chacun des membres du ménage " un tel (une telle) est-il (elle) handicapée(e) ou a-t-il (elle) quelques gênes ou difficultés dans la vie quotidienne? »"

La densité de médecins de secteur 2 de la zone d'études et d'aménagement du territoire (Zeat) a été introduite afin de prendre en compte les caractéristiques de l'offre de soins, et en particulier les prix des soins auxquels font face les ménages, qui sont des déterminants de la demande d'assurance (Legal 2009).

Afin d'étudier l'influence du revenu sur la demande de complémentaire santé, le revenu disponible du ménage par unité de consommation a été introduit dans les deux équations en quintiles calculés sur la distribution pondérée des revenus de la population des ménages non éligibles à la CMU-C. Le revenu disponible du ménage a été calculé à partir du montant mensuel total en euros de l'ensemble des ressources des ménages hors ressources exceptionnelles auquel sont retranchés les impôts (impôts sur le revenu, impôts et taxes de la résidence principale, impôts et taxes de la résidence secondaire ou autre logement, taxes automobiles, autres impôts et taxes). Pour tenir 
Plusieurs résultats suggèrent ensuite que la demande de complémentaire santé résulte d'un arbitrage entre le risque perçu de dépenses de santé et le prix des contrats. De manière cohérente avec l'augmentation des dépenses de santé et des primes avec l'âge, les dépenses de complémentaire croissent de manière continue avec l'âge. La relation existant entre l'âge et la propension d'être couvert est en revanche moins triviale. En cohérence avec les résultats de Saliba et Ventelou (2007), c'est parmi les plus jeunes que la propension à souscrire un contrat de complémentaire santé est la plus faible, du fait en particulier de la perte du bénéfice de la couverture complémentaire de leurs parents, de revenus plus faibles et d'une meilleure santé en moyenne. La probabilité d'être couvert croît ensuite régulièrement jusqu'à l'âge de 60 ans, avant d'être à nouveau significativement plus réduite parmi la population âgée de plus de 80 ans. Cette moindre probabilité d'être couvert peut tout d'abord s'expliquer par la période de transition entre la perte de la couverture subventionnée par l'employeur au moment du passage à la retraite, et la souscription à un contrat individuel. De plus, aux âges les plus élevés, le bénéfice attendu de la couverture complémentaire peut en outre être réduit dans le cas d'une prise en charge à $100 \%$ par l'Assurance maladie des dépenses de santé liées à une «Affection de longue durée » (ALD).

Alors que l'état de santé des membres de la famille devrait être un indicateur des dépenses de santé anticipées du ménage, l'analyse ne révèle aucune association significative entre la présence d'au moins une personne dans le ménage souffrant d'un handicap ou de difficultés dans la vie quotidienne en raison d'un problème de santé et le montant des dépenses. Cette absence de corrélation peut alors s'expliquer par une forte corrélation entre cette mesure de l'état de santé et le statut d'ALD. Néanmoins, même après contrôle par le statut d'ALD, l'analyse de Saliba et Ventelou (2007) n'avait pas établi non plus de lien entre état de santé et probabilité d'être couvert. Notre analyse montre, en revanche, que les dépenses de complémentaire santé sont sensiblement plus élevées dans les ménages dont au moins l'un des membres présente un handicap ou des difficultés dans la vie quotidienne en raison d'un problème de santé. En dépit d'une mesure relativement grossière de l'état de santé, en comparaison des indicateurs disponibles dans les enquêtes santé, l'analyse permet donc de mettre en relation la demande de complémentaire santé avec le niveau des besoins de soins du ménage.

Du point de vue des types de ménages, les couples (avec ou sans enfants) s'opposent nettement aux personnes seules (avec ou sans enfants), ces derniers détenant moins souvent une couverture complémentaire. Ce résultat est en partie dû à notre définition d'un ménage couvert : il suffit en effet que l'un des deux membres du couple soit couvert pour que le ménage soit considéré comme couvert. Mais cette explication est à prendre avec précaution puisque les " autres ménages » ont une probabilité plus faible d'être couverts alors qu'ils sont composés par définition de plusieurs adultes. Les dépenses de complémentaire santé diminuent quant à elles avec la taille de la famille, suggérant ainsi la décroissance du coût par tête de contrats avec le nombre de personnes couvertes. Cette évolution du prix par tête des contrats peut être également une explication du plus grand taux de couverture des couples.

\section{Encadré 4 (suite)}

compte de la taille des ménages, le revenu disponible par unité de consommation a été calculé en divisant le revenu total par le nombre d'unités de consommation défini selon l'échelle d'équivalence de l'OCDE qui attribue un poids de 1 à la première personne du ménage, un poids de 0,5 aux autres membres du ménage âgés de 14 ans et plus et un poids de 0,3 aux enfants âgés de moins de 14 ans.

Plusieurs autres indicateurs ont été retenus pour caractériser le statut sociodémographique des ménages : l'âge du chef de ménage, son niveau d'éducation (défini par le plus haut diplôme obtenu), sa catégorie professionnelle et son statut d'occupation, le type de ménage.

Enfin, le sexe du chef de ménage a été introduit dans le vecteur $X_{1 i}^{*}$ uniquement, jouant ainsi le rôle de variable d'exclusion, cette dimension n'étant associée significativement qu'à la propension à se couvrir individuellement.

L'exclusion de l'analyse des ménages couverts par le biais de leur employeur peut induire un biais de sélection. Nous avons cependant renoncé à traiter ce biais en raison de l'absence dans l'enquête étudiée de variable(s) identifiante(s) satisfaisante(s), telles que le secteur d'activité. La validité des résultats de l'estimation des dépenses de complémentaire santé est donc limitée à la souspopulation couverte à titre individuel, mais ne rend pas compte des dépenses qui seraient engagées par les ménages couverts par leur employeur s'ils ne l'étaient pas. 
Tableau 3

Déterminants de la couverture par une complémentaire santé et des dépenses de complémentaire santé par tête engagées en 2006

\begin{tabular}{|c|c|c|c|c|c|c|}
\hline \multirow{2}{*}{ Variables } & \multicolumn{3}{|c|}{ Probabilité de se couvrir } & \multicolumn{3}{|c|}{ Dépenses par tête } \\
\hline & \multicolumn{2}{|c|}{ Coefficient } & Écart-type & \multicolumn{2}{|c|}{ Coefficient } & Écart-type \\
\hline \multicolumn{7}{|l|}{ Revenu par unité de consommation } \\
\hline Premier quintile & Ref. & & & Ref. & & \\
\hline Deuxième quintile & 0,1799 & $(* \star *)$ & 0,0599 & $-0,0316$ & & 0,0291 \\
\hline Troisième quintile & 0,2113 & $(* \star *)$ & 0,0628 & 0,0377 & & 0,0306 \\
\hline Quatrième quintile & 0,3626 & $\left({ }^{* \star *}\right)$ & 0,3626 & 0,0277 & & 0,0365 \\
\hline Cinquième quintile & 0,3133 & $(* \star *)$ & 0,0745 & 0,1517 & $\left({ }^{\star \star \star}\right)$ & 0,0365 \\
\hline \multicolumn{7}{|l|}{ Situation par rapport au travail } \\
\hline Occupe un emploi & Ref. & & & Ref. & & \\
\hline étudiant, stagiaire & $-0,5752$ & $\left({ }^{* \star *}\right)$ & 0,1727 & $-0,2170$ & $\left({ }^{\star}\right)$ & 0,1315 \\
\hline Chômeur & $-0,3614$ & $\left({ }^{* \star *}\right)$ & 0,093 & 0,1066 & $\left({ }^{\star}\right)$ & 0,0597 \\
\hline Retraité & 0,4549 & $\left({ }^{\star \star \star}\right)$ & 0,1097 & 0,0695 & & 0,0468 \\
\hline Femme ou homme au foyer & 0,0788 & & 0,2067 & 0,0289 & & 0,0961 \\
\hline Autres & 0,3261 & & 0,2001 & $-0,0922$ & & 0,0861 \\
\hline \multicolumn{7}{|l|}{ Catégorie socio-profesionnelle } \\
\hline Agriculteur & 0,3752 & $\left({ }^{\star \star \star}\right)$ & 0,1185 & 0,2111 & $\left({ }^{\star \star \star}\right)$ & 0,0459 \\
\hline Artisan-commerçant & $-0,1455$ & $\left({ }^{*}\right)$ & 0,0821 & 0,0955 & $\left({ }^{* \star *}\right)$ & 0,0361 \\
\hline Cadre et prof,intell,supérieure & 0,0072 & & 0,0867 & 0,0550 & & 0,0346 \\
\hline Profession intermédiaire & 0,1212 & $\left({ }^{\star}\right)$ & 0,0699 & $-0,0497$ & $\left({ }^{*}\right)$ & 0,0291 \\
\hline Employé & 0,0577 & & 0,0645 & 0,0010 & & 0,0273 \\
\hline Ouvrier & Ref. & & & Ref. & & \\
\hline Sans profession & $-0,1565$ & & 0,1487 & 0,0376 & & 0,0689 \\
\hline \multicolumn{7}{|l|}{ Niveau d'instruction } \\
\hline Aucun diplôme & $-0,3240$ & $\left({ }^{* \star \star}\right)$ & 0,064 & $-0,0299$ & & 0,0339 \\
\hline CEP & $-0,1216$ & & 0,0743 & 0,0405 & & 0,0279 \\
\hline $1^{\text {er }}$ cycle CAP/BEP & Ref. & & & Ref. & & \\
\hline 2nd cycle & $-0,1258$ & $\left({ }^{*}\right)$ & 0,0698 & 0,1011 & $\left({ }^{\star \star \star}\right)$ & 0,0288 \\
\hline Supérieur & $-0,1279$ & $\left({ }^{*}\right)$ & 0,0677 & 0,0469 & $\left({ }^{\star}\right)$ & 0,0276 \\
\hline Sexe & Ref. & & & & & \\
\hline Femme & 0,2548 & $\left({ }^{\star \star \star}\right)$ & 0,0526 & & & \\
\hline \multicolumn{7}{|l|}{ Age } \\
\hline $18-29$ ans & $-0,3660$ & $\left({ }^{\star \star \star}\right)$ & 0,0827 & $-0,4141$ & $\left({ }^{\star \star \star}\right)$ & 0,0487 \\
\hline $30-39$ ans & $-0,0930$ & & 0,0716 & $-0,3389$ & $\left({ }^{\star \star \star}\right)$ & 0,0313 \\
\hline $40-49$ ans & $-0,1169$ & $\left({ }^{\star}\right)$ & 0,0699 & $-0,2050$ & $\left({ }^{\star \star \star}\right)$ & 0,0312 \\
\hline $50-59$ ans & Ref. & & & Ref. & & \\
\hline $60-69$ ans & $-0,0723$ & & 0,1109 & 0,1163 & $\left({ }^{\star \star \star}\right)$ & 0,0405 \\
\hline $70-79$ ans & $-0,0742$ & & 0,1213 & 0,1034 & $\left({ }^{\star \star}\right)$ & 0,0434 \\
\hline 80 ans et + & $-0,2710$ & $(* *)$ & 0,1344 & 0,1518 & $\left({ }^{\star \star \star}\right)$ & 0,0513 \\
\hline \multicolumn{7}{|l|}{ Limitations fonctionnelles } \\
\hline au moins 1 personne & 0,0132 & & 0,0508 & 0,0454 & $\left({ }^{\star *}\right)$ & 0,0197 \\
\hline \multicolumn{7}{|l|}{ Type de ménage } \\
\hline Personne seule & $-0,3502$ & $\left({ }^{\star \star \star}\right)$ & 0,0627 & 0,6130 & $\left({ }^{\star \star \star}\right)$ & 0,0312 \\
\hline Famille monoparentale & $-0,4324$ & $(* \star \star)$ & 0,0839 & 0,1206 & $\left({ }^{\star \star \star}\right)$ & 0,0432 \\
\hline Couple sans enfant & 0,0040 & & 0,0634 & 0,0753 & $(* \star \star)$ & 0,0255 \\
\hline Couple avec enfant & Ref. & & & Ref. & & \\
\hline Autres & $-0,2813$ & $(* \star)$ & 0,1181 & $-0,0577$ & & 0,0562 \\
\hline \multicolumn{7}{|l|}{ Densité de médecins secteur 2 par } \\
\hline$<16 \%$ & 0,0807 & & 0,0494 & $-0,0050$ & & 0,0199 \\
\hline [16 \%-24\%] & Ref. & & & Ref. & & \\
\hline$>24 \%$ & $-0,0972$ & $\left({ }^{\star}\right)$ & 0,0497 & 0,0594 & $\left({ }^{\star \star \star}\right)$ & 0,0216 \\
\hline Ratio de Mills & - & & & $-0,4026$ & $\left({ }^{*}\right)$ & 0,2145 \\
\hline Taille de l'échantillon & 6916 & & & 6043 & & \\
\hline
\end{tabular}

Lecture : les variables expliquées sont la probabilité d'être couvert par une complémentaire et le logarithme des dépenses par tête. ${ }^{* * *}$ : significatif au seuil de $1 \% ;{ }^{* *}$ : significatif au seuil de $5 \% ;{ }^{*}$ : significatif au seuil de $10 \%$.

Champ : Ménages enquêtés en France métropolitaine dont la personne de référence est âgée de 18 ans et plus et non éligibles à la $C M U-C$, et couverts par une complémentaire santé à titre individuel uniquement (équation 2), ainsi que les non couverts (équation 1).

Source : Enquête Budget de Famille 2006. 
Enfin, les ménages vivant dans des zones où la densité de médecins en secteur 2 pratiquant des dépassements d'honoraires est plus élevée, comme l'Ile-de-France, ont à la fois une probabilité plus faible d'être couvert mais des dépenses de complémentaire plus élevées. Le montant des dépassements peut désinciter les ménages à se couvrir, lorsqu'ils n'ont les moyens que d'acquérir des contrats de moindre qualité, et au contraire à choisir des contrats plus couvrants dès qu'ils le peuvent.

L'ensemble de ces résultats suggèrent que la demande de complémentaire santé peut se comprendre par un arbitrage rationnel, fonction des risques pesant sur les ménages ainsi que des prix des contrats. Cependant, c'est avant tout un choix fortement contraint par le revenu. La persistance de personnes non couvertes ou faiblement couvertes en France semble avant tout expliquée par les difficultés financières des ménages les plus modestes.

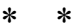

Cet article se proposait d'éclairer pour la première fois en France la question du taux de recours à une complémentaire santé en France et ses déterminants à partir du relevé exhaustif des dépenses des ménages, tel que renseigné dans l'enquête Budget de Famille 2006.

Ses résultats confirment tout d'abord les résultats des études précédentes tant sur le bilan quantitatif du taux de recours à une complémentaire santé que sur les déterminants du recours à une complémentaire santé. Environ 9 personnes sur 10 sont couvertes par une assurance complémentaire en France et le taux de couverture varie fortement avec le niveau de vie des ménages. Ainsi, le taux de non couverture par une complémentaire santé décroît de $19 \%$ parmi les ménages appartenant au premier décile de revenu à moins de $8 \%$ pour les ménages les plus aisés. Le rôle essentiel du revenu est par ailleurs confirmé par les résultats de l'analyse économétrique des déterminants du recours à la complémentaire santé et des dépenses engagées pour son achat.

Le relevé des dépenses engagées pour l'achat d'une complémentaire santé nous a également permis d'apprécier la part des ressources consacrées par les ménages pour l'achat d'une complémentaire santé ainsi que son accessibilité financière. Le montant des dépenses consacrées augmente fortement avec le niveau de revenu. Pour autant, parmi les ménages couverts, les plus modestes consacrent 8,5\% de leur revenu disponible à l'acquisition d'un contrat de couverture complémentaire lorsqu'ils sont couverts par un contrat individuel et $10,7 \%$ lorsqu'ils sont couverts par le biais de leur employeur, contre seulement respectivement $2,3 \%$ et $3,3 \%$ pour les plus riches. Ainsi l'assurance complémentaire peut être jugée en 2006 comme non accessible financièrement à 18,5\% de la population française, et encore à 10,3\% de la population non couverte par la CMU-C, dans la mesure où l'achat d'un contrat de complémentaire santé les conduit à tomber en dessous du seuil de pauvreté.

Ces résultats invitent à approfondir l'étude des liens entre couverture complémentaire santé et recours aux soins pour les populations pauvres afin d'évaluer en termes de santé publique l'efficacité du financement du système de santé.

\section{BIBLIOGRAPHIE}

Arnould M-L et Vidal G. (2008), « Typologie des contrats les plus souscrits auprès des complémentaires en 2006 », Études et Résultats, nº 663.

Auerbach D. et Ohri S. (2006), « Price and the Demand for Non-Group Health Insurance ", Inquiry, vol. 43, pp. 122-134.

Barsky R.B., Juster F.T., Kimball M.S. et Shapiro M.D. (1997), « Preference Parameters and Behavioral Heterogeneity : An Experimental Approach to the Health and Retirement Survey »,
Quarterly Journal of Economics, vol. 112, $\mathrm{n}^{\circ} 2$, pp. 537-580.

Boisguérin B. (2007), « Les bénéficiaires de la CMU au 31 décembre 2005 », Études et Résultats, $\mathrm{n}^{\circ} 512$.

Boisguérin B., Després C., Dourgnon P., Fantin R. et Legal R. (2010), «Étudier l'accès aux soins des assurés CMU-C, une approche par le renoncement aux soins », dans Santé, soins et protection sociale en 2008, Irdes 2010/06, pp. 31-40. 
Buchmueller T., Couffinhal A., Grignon M. et Perronnin M. (2004), «Access to physician services : does supplemental insurance matter? Evidence from France ", Health Economics, vol. $13, \mathrm{n}^{\mathrm{o}} 7$, pp. 669-687.

Bundorf K. M et Pauly M. V. (2006), « Is health insurance affordable for the uninsured ? », Journal of Health Economics, vol. 25, no 4, pp. 650-673.

Couffinhal A., Perronnin M, Chevalier J. et Lengagne P. (2004), « Accès à la couverture complémentaire maladie en France : une comparison des niveaux de remoursements - Enquêtes ESPS 2000 et 2002 », Questions d'économie de la santé, nº 80.

Doiron D., Jones G. et Savage E. (2008), " Healthy, Wealthy and Insured ? The Role of Self-Assessed Health in the Demand for Private Health Insurance », Health Economics, vol. 17, no 3, pp. 317-334.

Dufour-Kippelen S., Legal A. et Wittwer J. (2006), « Comprendre les causes du non-recours à la CMUC », Rapport Legos pour le Fonds Cmu, http://www.cmu.fr/userdocs/Non-recours LEGOS.pdf.

Fenina A. et Geoffroy Y. (2007), « Les Comptes nationaux de la santé en 2006 », Études et Résultats, $\mathrm{n}^{\circ} 593$.

Fenina A., Le Garrec M-A et Koubi M. (2011), «Les Comptes nationaux de la santé en 2010 », Études et Résultats, $\mathrm{n}^{\mathrm{o}} 773$.

Fonds CMU (2008), « L'aide complémentaire santé en 2007 », Rapport du Fonds CMU au Gouvernement sur l'évolution du prix et $d u$ contenu des contrats ayant ouvert droit à l'aide complémentaire santé en 2007.

Fonds CMU (2011), Rapport d'activité $2010 d u$ Fonds CMU, 22 juin 2011, http://www.cmu.fr/ userdocs/RA_2010.pdf

Goutard L. et Pujol J. (2008), « Les niveaux de vie en 2006 », Insee Première, Insee, nº 1203.

Grignon M. et Kambia-Chopin B. (2009), " Income and the demand for complementary health insurance in France », Document de travail de l'Irdes, $\mathrm{DT}^{\circ} 24$.

Grignon M. et Kambia-Chopin B. (2010), «Quelle subvention optimale pour l'achat d'une complémentaire santé ? », Questions d'économie de la Santé, Irdes, $\mathrm{n}^{\circ} 153$.
Grossman M. (2000), " The human capital model ", dans Culyer, A.J. and J.P. Newhouse (eds), Handbook of health economics, pp. 347408, Elsevier, Amsterdam.

Guillaume S. et Rochereau T. (2010), « La protection sociale complémentaire collective : des situations diverses selon les entreprises ", Questions d'économie de la Santé, Irdes, nº 155.

Jusot F. et Wittwer J. (2009), « L'accès financier aux soins en France : bilan et perspective ", Regards croisés sur l'économie, vol. 5, $\mathrm{n}^{\circ} 1$, pp. 102-109.

Guthmuller S., Jusot F., Wittwer J. et Desprès C. (2012), « Le Recours à l'Aide complémentaire santé : les enseignements d'une expérimentation sociale à Lille ", Économie et Statistique, à paraître.

Kambia-Chopin B., Perronnin M., Pierre A. et Rochereau T. (2008a), « La complémentaire santé en France en 2006 : un accès qui reste inégalitaire. Résultats de l'enquête Santé Protection Sociale 2006 (ESPS 2006) », Questions d'économie de la Santé, Irdes, no 132.

Kambia-Chopin B., Perronnin M., Pierre A. et Rochereau T. (2008b), « Les contrats complémentaires individuels : quel poids dans le budget des ménages ? » dans Enquête sur la Santé et la protection sociale 2006 (ESPS 2006), Rapport Irdes no 1701, pp. 43-55.

Legal, R. (2009), « L'influence de l'offre de soins et du niveau des primes sur la demande d'assurance complémentaire santé en France », Revue Économique, $\mathrm{n}^{\circ}$ 60, pp. 441-53.

Marical F. et Saint-Pol (de) T. (2007), « La complémentaire santé : une généralisation qui n'efface pas les inégalités », Insee Première, Insee, nº 1142.

Marquis M.S. et Long S.H. (1995), « Worker Demand for Health Insurance in the Non-Group Market », Journal of Health Economics, vol. 14, $\mathrm{n}^{\mathrm{o}} 1$, pp. 47-63.

Monheit A. et Vistnes J. (2008),. « Health Insurance Enrollment Decisions : Preferences for Coverage, Worker Sorting, and Insurance Take $\mathrm{Up} »$, Inquiry, vol. 45, n 2, pp. 153-67.

Perronnin M., Pierre A. et Rochereau T. (2011), « La complémentaire santé en France en 2008 : une large diffusion mais des inégalités d'accès », Questions d'économie de la Santé, Irdes, nº 161. 
Raynaud D. (2005), « Les déterminants individuels des dépenses de santé : l'influence de la catégorie sociale et de l'assurance maladie complémentaire », Études et résultats, nº 378.
Saliba B. et Ventelou B. (2007), « Complementary health insurance in France. Who pays ? Why? Who will suffer from public disengagement? ", Health Policy, $\mathrm{n}^{\circ}$ 81, pp. 166-182. 